\title{
Holmium laser enucleation of prostate by using en-bloc and bladder neck preservation technique: technical consideration and influence on functional outcomes
}

\author{
Pu Li, Chengming Wang, Min Tang, Peng Han, Xiaoxin Meng \\ Department of Urology, The First Affiliated Hospital of Nanjing Medical University, Nanjing, China \\ Contributions: (I) Conception and design: X Meng, P Li; (II) Administrative support: X Meng; (III) Provision of study materials or patients: X Meng, \\ P Li; (IV) Collection and assembly of data: P Han, C Wang; (V) Data analysis and interpretation: P Li, M Tang, X Meng; (VI) Manuscript writing: \\ All authors; (VII) Final approval of manuscript: All authors. \\ Correspondence to: Xiaoxin Meng, MD, PhD. Department of Urology, The First Affiliated Hospital of Nanjing Medical University, 300 Guangzhou \\ Road, Nanjing 210029, China. Email: mengxiaoxin1304@163.com.
}

Background: This study aimed to evaluate the efficacy and safety of holmium laser enucleation of prostate (HoLEP) by using en-bloc and bladder neck preservation technique on benign prostatic hyperplasia (BPH) and assess the influence of this procedure on urinary and sexual functional outcomes.

Methods: A total of 704 patients with BPH undergoing HoLEP were retrospectively analysed. Amongst them, 213 who met the sexual function assessment criteria were further assessed the influence of the technique on sexual functional outcomes.

Results: The mean operative time, resected prostate weight and mean estimated blood loss were $69.3 \pm 14.8$ $\min , 53.4 \pm 17.6 \mathrm{~g}$ and $62.1 \pm 23.7 \mathrm{~mL}$, respectively. The mean duration of catheterization after surgery and mean hospital stay were $2.1 \pm 1$ and $5.4 \pm 3.6$ days, respectively. The post-void residual volume, maximum urinary flow rate, international prostate symptom score and quality of life score were all significantly improved postoperation $(\mathrm{P}<0.05)$. Amongst the 213 patients with normal antegrade ejaculation before surgery, postoperative international index of erectile function scores slightly improved, although the difference was insignificant $(\mathrm{P}>0.05)$. The difference in the Erection Hardness Grading Scale before and after surgery was statistically insignificant $(\mathrm{P}>0.05)$. A total of $25(11.7 \%)$ patients had retrograde ejaculation after surgery.

Conclusions: HoLEP by an en-bloc and bladder neck preservation technique can obtain good functional outcome with small injury and high efficiency, especially for patients who need to preserve normal sexual function and anterograde ejaculation.

Keywords: Holmium laser enucleation of prostate; en-bloc; bladder neck preservation; sexual function; retrograde ejaculation

Submitted Apr 08, 2020. Accepted for publication Oct 16, 2020.

doi: $10.21037 /$ tau-20-852

View this article at: http://dx.doi.org/10.21037/tau-20-852

\section{Introduction}

Benign prostatic hyperplasia (BPH) and its associated lower urinary tract symptoms (LUTS) are extremely common amongst aged men. BPH occurs in approximately $50 \%$ of men in their $50 \mathrm{~s}$ and $80 \%$ of men by the eighth decade of life (1), thereby representing a common health problem affecting males. Although the medical management of LUTS due to BPH is becoming increasingly effective, especially for the widespread use of $\alpha$-blockers, minimally invasive and surgical treatments remain common. Surgical treatment is still considered as the ultimate treatment for refractory and severe BPH-associated LUTS or when 
patients have side effects or poor tolerance to medical options.

For relatively young patients with $\mathrm{BPH}$, the main outcomes of treatment are not only improvement in an international prostate symptom score (IPSS) which represents ideal urinary function but also the preservation of sexual function (2). The transurethral resection of the prostate (TURP) is the gold standard for the surgical treatment of $\mathrm{BPH}$ for several decades. However, the status of TURP has been increasingly challenged due to the increased incidence of complications.

Several new minimally invasive techniques have been developed to treat BPH/LUTS, including anatomical endoscopic enucleation of the prostate (AEEP), which first performed as holmium laser enucleation of prostate (HoLEP) (3). Improvements in technique and equipments have led to true anatomical enucleation of prostate of any size (4). When compared with TURP, currently the reference gold standard, patients undergoing AEEP benefit from a shorter catheterization time, shorter hospital stay, and fewer complications (5). Precisely because the welldocumented superiority over the traditional therapies, including open surgery, TURP, and other plasma and laser modalities, HoLEP is widespread implemented. These benefits make HoLEP the procedure of choice for men seeking surgical relief for BPH related LUTS. Many authors believe that HoLEP is a promising alternative to TURP. However, the problem remains. Dose there still exist potential improvement for HoLEP technique? What the benefits HoLEP can offer to $\mathrm{BPH}$ patients over traditional TURP?

The purpose of this study was to investigate the efficacy and safety of HoLEP by an en-bloc and bladder neck preservation technique in treating $\mathrm{BPH}$ and detect the effect of this technique in the prevention of male sexual function. We present the following article in accordance with the STROBE reporting checklist (available at http:// dx.doi.org/10.21037/tau-20-852).

\section{Methods}

\section{Patient identification and data collection}

Patients who underwent HoLEP between July 2013 and May 2018 in the First Affiliated Hospital of Nanjing Medical University were retrospectively analysed. This study was conducted in accordance with the Declaration of Helsinki (as revised in 2013) and approved by Institutional
Review Board (IRB) of the First Affiliated Hospital of Nanjing Medical University (registration ID 2013-SRFA051). Informed consent was obtained from all individual participants included in the study. All patients were initially investigated by verifying the medical history, physical examination and laboratory examination, including digital rectal examination, prostate-specific antigen (PSA) level determination, uroflowmetry testing or urodynamic analysis and prostate volume measurement by ultrasonography or MRI, as well as post-void residual (PVR) volume, maximum urinary flow rate (Qmax,), IPSS and QoL score determination. The inclusion criteria were IPSS $\geq 8$, Qmax $\leq 15 \mathrm{~mL} / \mathrm{s}, \mathrm{PVR} \geq 50 \mathrm{~mL}$, or QoL score $\geq 3$. Patients with prostate cancer as confirmed by biopsy or postoperative pathology, bladder outlet obstruction due to urethral stricture and severe detrusor dysfunction were excluded. Patients with incomplete surgical data or followup information were also excluded. A total of 704 patients who underwent HoLEP surgery met the requirements. We further screened patients who were included in the present sexual function assessment study. Patients included in the sexual function assessment group should satisfy the following criteria: (I) patient with a partner and performs sexual activity; (II) that with the International Index of Erectile Function (IIEF-5) score of $\geq 12$; (III) that with normal antegrade ejaculation without ejaculation pain; (IV) that who can cooperate with the investigation. Finally, 213 patients were included in the sexual function study.

\section{Surgical procedure}

All HoLEP procedures were performed under general anaesthesia by the same consulting surgeon (MXX), who had $>1,000$ surgical cases before this study was carried out. The HoLEP equipment included a high-power 100W Ho:YAG laser (Versa Pulse Select; Lumenis Inc., Yokneam, Israel), a 550- $\mu \mathrm{m}$ end fire optic fiber (SlimLine 550; Lumenis Inc.), a modified 26-Fr Storz continuous-flow resectoscope with a working element for stabilizing the laser fiber, a Storz rigid nephroscope, and a tissue morcellator (VersaCut system; Lumenis Inc.). HoLEP was performed as previously described (6). In brief, the $26 \mathrm{Fr}$ continuousflow resectoscope with a $550-\mu \mathrm{m}$ optic fibre was inserted into the urethra under direct vision. After the urethra and bladder were inspected, anatomic markers were located. Resection was performed at an energy of 80 Watt (2.0 Joule at $40 \mathrm{~Hz}$ ), beginning in either side at the level proximal to 
the verumontanum. Resection was performed at an energy of 80 Watt (2.0 Joule at $40 \mathrm{~Hz}$ ), beginning in either side at the level proximal to the verumontanum. The first incision was made around the verumontanum in a reversed $U$ shape. After initial resection was performed, an anatomic plane was formed to reach the surgical capsule, partially raise the middle lobe, and mechanically develop and mobilize both side lobes apically. This step was then continued until a mucosal strip was seen on both side lobes. After that the mucosal strip between hyperplastic gland and external sphincter was cut off, which allowed complete mobilization of the apical part of prostate. Subsequently, with the shaft of resectoscope and laser impulse, we developed the plane bilaterally between the surgical capsule and hyperplastic gland. The plane was converged centrally at anterior commissure. Dissection was then continued towards the bladder neck by laser cutting and mechanical methods. Then a 'channel' between the surgical capsule and the anterior commissure was created approach to the bladder neck at 12 o'clock. Once the surgical preparation reached the bladder neck, an transect incision was made by the laser to entered into the bladder lumen. This incision was then continued to both sides. At this step, some white and shiny circular fibers could be seen at $0.5 \mathrm{~cm}$ from the bladder neck, which were visually different from hyperplastic gland. This is exactly the bladder neck and internal sphincter that need to be preserved. Then both side lobes were dissociated from the bladder neck. The lateral sides of both side lobes were then circularly mobilized until the lateral part of the middle lobe on both sides was reached. After this, the middle lobe was mobilized retrogradely to the level of bladder neck. The hyperplastic gland was then pushed into the bladder lumen (Video 1). The preserved bladder neck looked like a 'dam'at 6 o'clock (Figure 1). After resection was performed, all lobes dropped into the bladder. A morcellator (Lumenis) with an off-set nephroscopic lens (Karl-Storz) was introduced, and prostate adenoma was morcellated under direct vision. Tissue shivers were removed by irrigation.

\section{Assessment methods}

Basic preoperative variables, including age at surgery, PSA level and prostate volume, were retrieved from patient records. Perioperative characteristics, such as operative time, estimated blood loss (EBL), duration of catheterization, hospital stay and complications, were also retrospectively obtained.
Functional recovery was assessed by evaluating related variables prior to surgery and at 3-, 6- and 12-month postoperation. Urinary outcome was evaluated in all 704 patients by using IPSS, QoL, PVR and Qmax. Urinary incontinence recovery was also used to assess urinary function outcomes. The severity of urinary incontinence was classified based on $24 \mathrm{~h}$ pad count (pads/day, PPD) and classified as mild (2 PPD), moderate (3-5 PPD) and severe ( $\geq 6 \mathrm{PPD})(7)$. Given that many patients amongst the 213 that were included in the sexual function study did not attempt to resume sexual activity at 3 months followup, sexual functional data, including erectile function and ejaculatory function, at 6 and 12 months postoperation were collected and compared with preoperative data.

Erectile function was measured using the five-item version of the IIEF-5 and erection hardness grading scale (EHGS) (8). According to the scale, grades $0,1,2,3$ and 4 represent 'not enlarged', 'larger but not hard', 'hard but not hard enough for penetration', 'hard enough for penetration but not completely hard' and 'completely hard and fully rigid', respectively. Ejaculatory function was evaluated based on the presence of ejaculation pain, change in semen volume and the presence of retrograde ejaculation. Change in semen volume and the presence of retrograde were subjectively measured by a self-assessment questionnaire, which contained questions as 'compared with preoperation, semen volume changes: increase, decrease, no change and no ejaculation'. The patients were required to select one of the options. If option 'no ejaculation' was selected, urine analysis and sperm detection will be carried out in the urine sample after ejaculation, to determine whether exist retrograde ejaculation.

\section{Statistical analysis}

The results of clinical parameters are shown as mean \pm standard deviation or number of cases (\%). Categorical and continuous data were analysed by Chi-squared test and paired $t$-test, respectively. Statistical data were analysed using IBM $^{\circledast}$ SPSS $^{\circledast}$ Statistics version 19.0. $\mathrm{P}<0.05$ were considered statistically significant. Cases with missing data or lack of follow-up information were excluded from this study.

\section{Results}

The preoperative demographics and basic clinical characteristics are shown in Table 1. HoLEPs by the en-bloc 

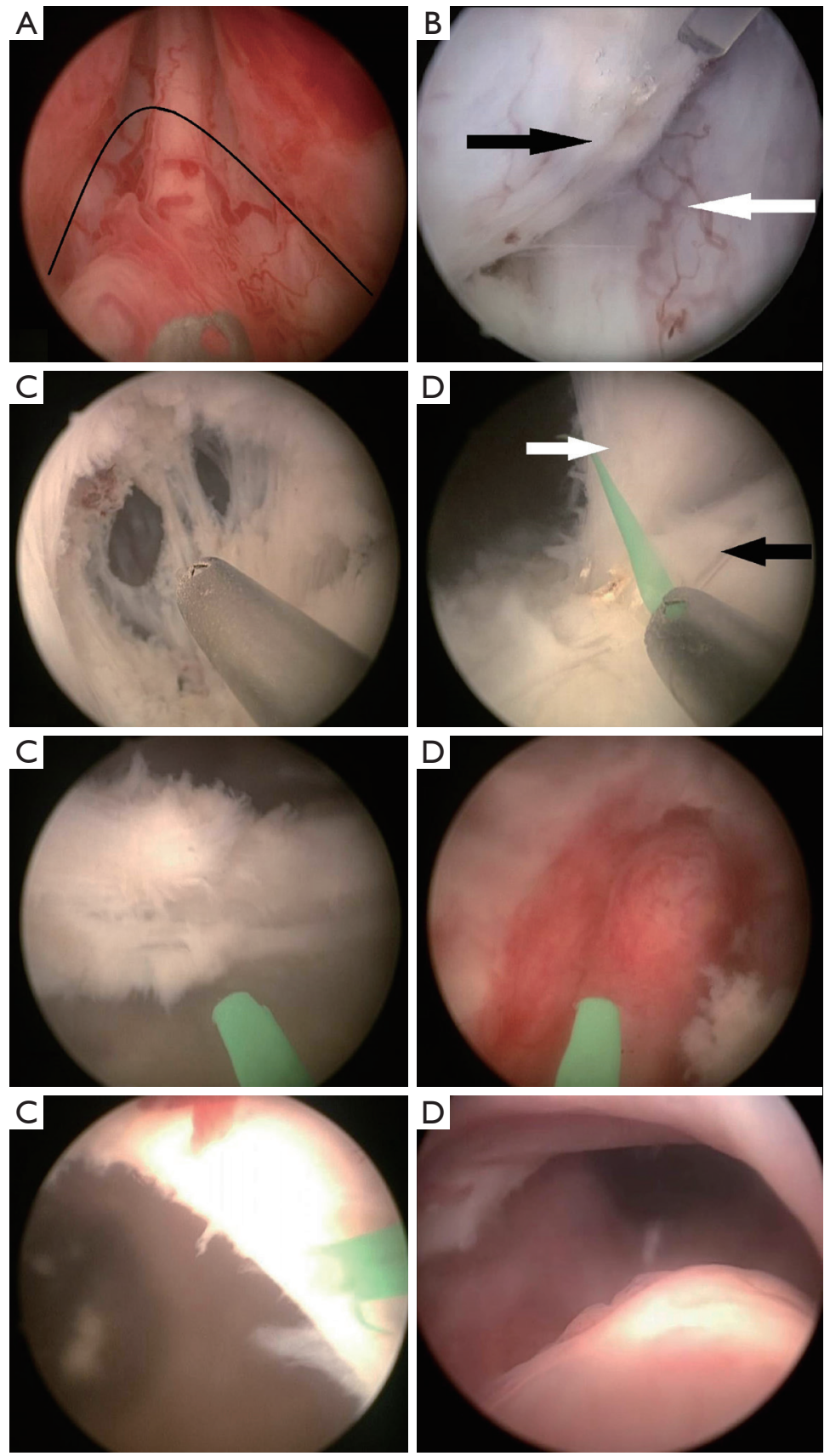

Figure 1 Holmium laser enucleation of prostate (HoLEP) procedure by an en-bloc and bladder neck preservation technique. (A) Image of verumontanum before operation. Initial resection was performed proximal to the verumontanum by using an inverted U-shaped incision. (B) An anatomic plane was formed between the surgical capsule and hyperplastic gland. The white and black arrows showed the surgical capsule and hyperplastic gland, respectively. (C) At 12 o'clock in the bladder neck. The hyperplastic gland was stripped from the bladder neck near 12 o'clock to cut into the bladder. Dense, whitish, ring-shaped internal sphincter tissue can be observed when hyperplastic gland was peeled off from the bladder neck. (D) The hyperplastic gland was continuously resected on either side from 12 o'clock, and the internal sphincter tissue of bladder neck was retained. The white and black arrows showed the bladder neck and hyperplastic gland, respectively. (E) A preserved bladder neck similar to a dam when observed from the six o'clock position. (F) A preserved verumontanum which was completely preserved to retain normal ejaculation. (G) An integrated external sphincter. The bladder neck and prostatic cavity can be observed at the external sphincter. (H) Image of cystoscopy at 3 months after surgery as observed at the verumontanum. 
Table 1 Baseline characteristics and perioperative variables for study participants

\begin{tabular}{lc}
\hline Variable & $\begin{array}{c}\text { Mean } \pm \text { SD or no. } \\
\text { of patients }(\%)\end{array}$ \\
\hline Number of cases $(\mathrm{n})$ & 704 \\
Preoperative & $67.8 \pm 6.9$ \\
Age (year) & $70.5 \pm 15.4$ \\
Prostate volume $(\mathrm{mL})$ & $3.2 \pm 2.6$ \\
Total PSA (ng/mL) & \\
Perioperative & $69.3 \pm 14.8$ \\
Operation time (min) & $62.1 \pm 23.7$ \\
EBL (mL) & $53.4 \pm 17.6$ \\
Resected prostate weight (g) & $0(0 \%)$ \\
Blood transfusion $(\mathrm{n})$ & $0(0 \%)$ \\
TURS (n) & $2.1 \pm 1.5$ \\
Duration of catheterisation (days) & $5.4 \pm 3.6$ \\
Hospital stay (days) &
\end{tabular}

SD, standard deviation; PSA, prostate specific antigen; EBL, estimated blood loss; TURS, transurethral resection syndrome.

and bladder neck preservation technique were technically successful in all 704 patients. The immediate postoperative outcomes were recorded. No major life-threatening complication was observed in the duration of hospital stay. The reoperation rate due to prolonged bleeding or clot retention was $1.6 \%$ (11/704). No transurethral resection syndrome was observed during and after HoLEP. The mean duration of catheterization after surgery was $2.1 \pm 1.5$ days, and the mean hospital stay was $5.4 \pm 3.6$ days. Furthermore, the rate of urinary retention after first catheter removal was $4.8 \%$ (34/704). All patients were discharged without catheter. After catheter removal, mild urinary incontinence was observed in 38 patients $(5.4 \%)$, while moderate incontinence was observed in 3 patients $(0.4 \%) .12$ patients (1.6\%) still had mild incontinence at 6 months follow-up, and 4 of them $(0.6 \%)$ had permanent mild incontinence until the last follow-up session (12 month after surgery). Two patients had anterior urethral stricture, and were resolved by urethral dilatation. No bladder neck contracture occurred.

Urinary function was measured on the based on PVR volume, Qmax, IPSS and QoL score. Compared with baseline variables before surgery, the PVR volume, Qmax, IPSS and QoL score were all significantly improved at 3-,
6- and 12 -month postoperation $(\mathrm{P}<0.05$, Table 2$)$. When 12 -month postoperation was considered as a timepoint to evaluate the improvement rate of urinary function, the IPSS showed a significant reduction of $74.8 \%$, the QoL score showed a significant reduction of $54.9 \%$, the PVR had a 76.8\% decrease, and Qmax had a 2.6-fold increase.

As shown in Table 3, regarding sexual function, the preoperative IIEF-5 and postoperative IIEF-5 scores at 6 and 12 months were $17.02 \pm 3.75$ and $17.33 \pm 2.04$ and $17.34 \pm 3.39$, respectively. Postoperative IIEF scores showed slight improvement compared with those at preoperation, although the difference was insignificant $(\mathrm{P}>0.05)$. Similarly, the difference in EHGS before and after surgery was statistically insignificant $(3.03 \pm 0.62,3.06 \pm 0.52,3.02 \pm 0.44$, $\mathrm{P}>0.05)$. Regarding ejaculation, the results were remarkable. All our patients did not experience ejaculation pain before and after surgery. Amongst the 213 patients with normal antegrade ejaculation before surgery, $25(11.7 \%)$ patients had retrograde ejaculation after surgery. For the 188 other patients who retained normal antegrade ejaculation, the semen volume after surgery decreased in $82(43.6 \%)$ patients but remained unchanged in $106(56.4 \%)$ patients.

\section{Discussion}

TURP is still considered as the gold standard for BPH surgery, but this procedure has many disadvantages, such as TUR syndrome, incomplete resection, high recurrence rate, haemorrhage, bladder neck contracture, urinary incontinence and damage to sexual function (9). However, TURP has been recognised for its role in improving the symptoms of LUTS. Traditional TURP minimally affects erectile function but can lead to retrograde ejaculation in $60-80 \%$ patients (10). Therefore, surgeons must be careful when treating young patients with normal sexual function and high requirement for postoperative sexual life are treated. The main reason for TURP damage to ejaculation is that the surgery destroys the integrity of the bladder neck and internal sphincter. The integrity of the bladder neck does not affect urinary fluency but can maintain a higher pressure at the bladder neck than that at the external sphincter during ejaculation, thereby excreting semen from the body (11). According to the above study, when the hyperplastic gland was resected during TURP, the bladder neck tissue was excised simultaneously, especially at 4 to 8 o'clock. This technique allowed the easy removal of the tissues that should be washed into the bladder. All of these procedures can keep the surgical field clear, minimise the 
Table 2 Pre- and postoperative urinary function results

\begin{tabular}{|c|c|c|c|c|}
\hline Variable & Preoperation & 3 months & 6 months & 12 months \\
\hline QoL (score) & $5.1 \pm 0.8$ & $2.4 \pm 0.7^{\star}$ & $2.2 \pm 0.8^{\star}$ & $2.3 \pm 0.6^{\star}$ \\
\hline PVR (mL) & $92.3 \pm 83.9$ & $22.5 \pm 15.6^{\star}$ & $25.7 \pm 16.8^{*}$ & $21.4 \pm 12.4^{*}$ \\
\hline $\mathrm{Qmax}(\mathrm{mL} / \mathrm{s})$ & $5.7 \pm 2.4$ & $19.2 \pm 3.7^{*}$ & $20.3 \pm 2.4^{*}$ & $20.6 \pm 3.8^{*}$ \\
\hline Mild & - & $38,5.4 \%$ & $12,1.6 \%$ & $4,0.6 \%$ \\
\hline Moderate & - & $3,0.4 \%$ & 0,0 & 0,0 \\
\hline Severe & - & 0,0 & 0,0 & 0,0 \\
\hline
\end{tabular}

IPSS, international prostatic symptomatic score; QoL, quality of life; PVR, post-void residual volume; and Qmax, maximum urinary flow rate. *, $t$-Test, compared with preoperative variables, $\mathrm{P}<0.05$.

Table 3 Pre- and postoperative sexual functional results

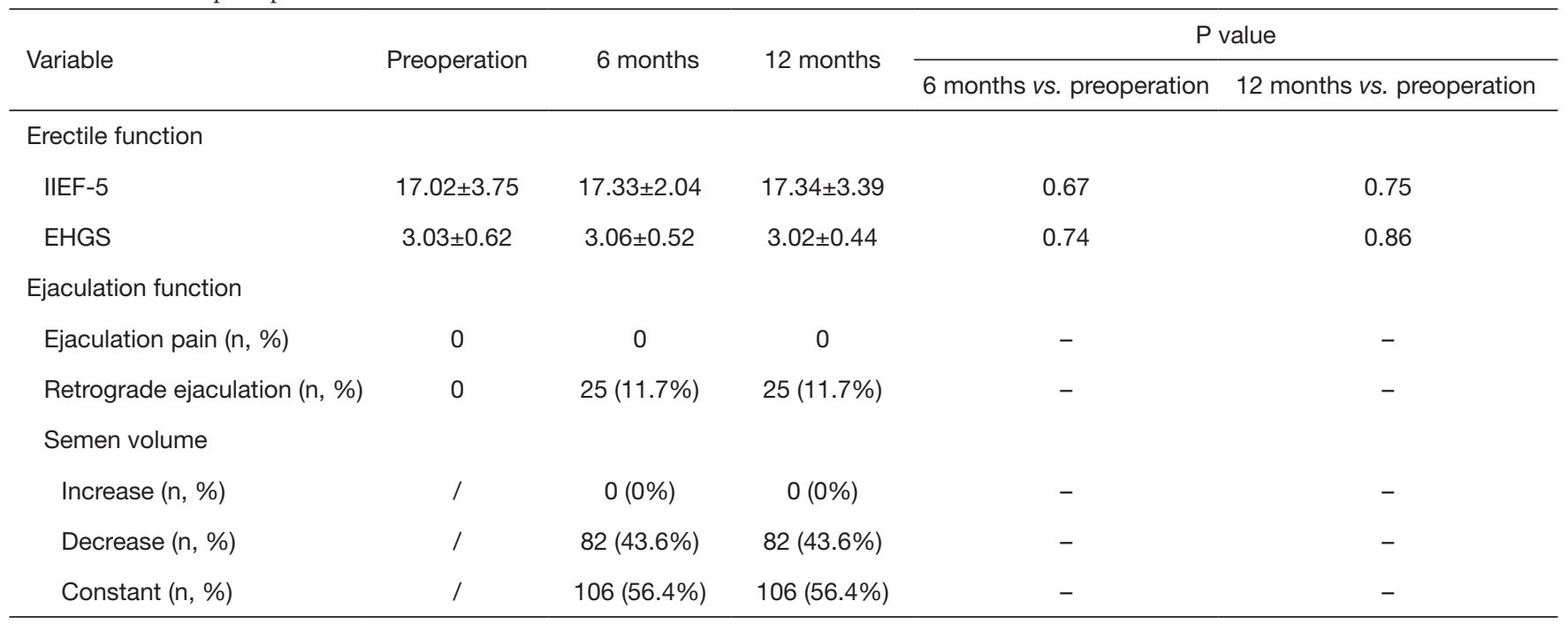

IIEF-5, five-item version of the International Index of Erectile Function; EHGS, Erection Hardness Grading Scale.

resistance of prostatic urethra and increase postoperative Qmax. However, this process damaged the bladder neck and increased postoperative retrograde ejaculation. Therefore, the normal anatomical structure of the bladder neck should be preserved whilst excising the hyperplastic gland.

Several novel minimally invasive treatment alternatives have demonstrated the ability to preserve postoperative sexual function to a better degree, all while providing relief of LUTS in an equally safe and efficacious manner $(12,13)$. Transurethral prostate enucleation techniques were developed to recapitulate the surgical dissection and tissue removal accomplished with open prostatectomy whilst maintaining the benefits realised with a transurethral approach. Transurethral enucleation techniques have been described with almost all energy sources. HoLEP representing the original and by far the most widely evaluated method. In addition to HoLEP, numerous other energy therapies exist for treatment of $\mathrm{BPH}$, including plasmakinetic enucleation of the prostate and Thulium laser enucleation of the prostate (ThuLEP). Few studies are available that directly compare HoLEP to these alternative modalities. Some studies showed that ThuLEP presents excellent vaporization and hemostatic capabilities with outcomes and complication rates similar to that of HoLEP (14). However, as a pulsed laser, HoLEP offers a "scar-free" feature on the prostatic surface and makes the 
plane of enucleation easy to develop and follow, providing superior visibility with precise incision and dissection, as well as greater versatility to the urologists: patients undergoing endoscopic surgery for BPH frequently require cystolitholapaxy, tumor resection or stricture ablation, all of which can be accomplished using the holmium laser (15).

The bladder neck is generally split during traditional HoLEP to achieve ideal urinary outcome. Similar to TURP, retrograde ejaculation is the most common sexual side effect after such HoLEP, with the incidence rates of approximately $70-80 \%$ (16-18). Bladder neck resection in HoLEP is the predominant factor contributing to high retrograde ejaculation rate (19).

When approaching to the bladder, a dense, whitish, circular braided bladder neck and internal sphincter tissue can be observed. Then, a bladder neck preservation technique was used. Tissues with the size of $0.5 \mathrm{~cm}$ from the bladder neck were retained, and only the mucosa was cut off from bladder neck. The retained bladder neck bulged at the 5 to 7 o'clock position, thereby completely retaining the original physiological functions of the internal urethral sphincter and bladder neck. This technique completely retained the bladder neck which can effectively reduce the occurrence of retrograde ejaculation and preserve urinary control function. No persistent and severe urinary incontinence was observed in this cohort, and normal antegrade ejaculation ability was retained in 80 patients amongst the 96 who had antegrade ejaculation before surgery.

The originally developed three-lobe and twolobe technique have popularized HoLEP. Then few modifications of the original technique have been developed, and several en-bloc enucleation methods have been introduced (20). During the en-bloc process, continuous incision and stripping were performed after the surgical capsule was exposed, thereby reducing unnecessary cutting and haemostasis and making morcellation easy. Thus, surgical efficiency is remarkably improved, and operation time is shortened.

The erectile nerve is distributed between the prostatic fascia and the levator ani fascia and relatively concentrated at the prostatic apex and below (21). During TURP, distal resection range generally does not exceed the verumontanum, wherein the cavernous nerve is concentrated. Generally, the depth of tissue damage caused by electric resection or vaporisation is limited. Thermal energy minimally affects the nerve if no perforation of surgical capsule occurs. This condition accounts for the minor change of erectile function before and after TURP operation (22).

Data regarding the effect of HoLEP on erectile function are conflicting. Whilst few studies have demonstrated a decrease in erectile function $(23,24)$, other studies have shown insignificant difference in EF following HoLEP (25). In the present study, postoperative IIEF and EHGS scores showed slight improvement compared with those at preoperation, although the differences were insignificant $(\mathrm{P}>0.05)$. These data showed that in most patients, HoLEP slightly helped in improving erectile function, at least did not cause significant negative impact. That was to say, HoLEP caused unremarkable damage to erectile function.

The blasting shock characteristic of holmium laser is used as the main mechanism in HoLEP which can retain intact prostate surgical capsule can lessen the damage to deep tissues. All these conditions can reduce the damage of erectile nerve. These advantages may be the reason why HoLEP is beneficial to erectile function in patients with BPH. HoLEP can also remove the additional volume of gland and minimise pressure on nerves and blood vessels that innervate erectile dysfunction. The necrosis of coagulation layer at the wound was also mild after HoLEP. The depth of coagulation plays an integral role in at least hemostasis and possibly other postoperative outcomes, for instance, a smaller coagulation layer leading to less urinary tract irritation and higher quality of life $(26,27)$, relieving the psychological burden of patients and enhancing their confidence in sexual function recovery.

In the present study, 82 (43.6\%) patients felt that semen volume decreased compared with that at preoperation. Prostatic fluid is an important component of semen. Semen volume reduction is related to the removal of hyperplastic glands. HoLEP is an anatomic enucleation, in which the amount of the removed tissue is significantly greater than that in TURP. The recurrence rate is decreased, but semen volume reduction also occurs. Although we attempted to preserve the intact bladder neck, the operation will still cause some damage to the structure of the internal sphincter. Therefore, a part of semen may be retrogradely ejaculated into the bladder in some patients.

The present study has a number of strengths, including a relatively large cohort of patients, a comprehensive preoperative investigation, and well validated assessments of patient sexuality. Likewise the study is not devoid of limitations, include its retrospective design, the mode of some subjective assessment used, and lack of parallel control group. 


\section{Conclusions}

HoLEP by an en-bloc technique has the advantages of low invasiveness, high efficiency and decreased blood loss and complications. The use of bladder neck preservation technique during surgery can effectively reduce the occurrence of retrograde ejaculation. The procedure is effective and safe for clinical application.

\section{Acknowledgments}

Funding: This study was funded by the National Natural Science Foundation of China (grant number 81302217).

\section{Footnote}

Reporting Checklist: The authors have completed the STROBE reporting checklist. Available at http://dx.doi. org/10.21037/tau-20-852

Data Sharing Statement: Available at http://dx.doi. org/10.21037/tau-20-852

Peer Review File: Available at http://dx.doi.org/10.21037/ tau-20-852

Conflicts of Interest: All authors have completed the ICMJE uniform disclosure form (available at http://dx.doi. org/10.21037/tau-20-852). The authors declare that they have no conflict of interest.

Etbical Statement: The authors are accountable for all aspects of the work in ensuring that questions related to the accuracy or integrity of any part of the work are appropriately investigated and resolved. This study was conducted in accordance with the Declaration of Helsinki (as revised in 2013) and approved by Institutional Review Board (IRB) of the First Affiliated Hospital of Nanjing Medical University (registration ID 2013-SRFA-051). Informed consent was obtained from all individual participants included in the study.

Open Access Statement: This is an Open Access article distributed in accordance with the Creative Commons Attribution-NonCommercial-NoDerivs 4.0 International License (CC BY-NC-ND 4.0), which permits the noncommercial replication and distribution of the article with the strict proviso that no changes or edits are made and the original work is properly cited (including links to both the formal publication through the relevant DOI and the license). See: https://creativecommons.org/licenses/by-nc-nd/4.0/.

\section{References}

1. Vuichoud C, Loughlin KR. Benign prostatic hyperplasia: epidemiology, economics and evaluation. Can J Urol 2015;22 Suppl 1:1-6.

2. Becher EF, McVary KT. Surgical Procedures for BPH/ LUTS: Impact on Male Sexual Health. Sex Med Rev 2014;2:47-55.

3. Pirola GM, Saredi G, Codas Duarte R, et al. Holmium laser versus thulium laser enucleation of the prostate: a matched-pair analysis from two centers. Ther Adv Urol 2018;10:223-33.

4. Michalak J, Tzou D, Funk J. HoLEP: the gold standard for the surgical management of BPH in the 21(st) Century. Am J Clin Exp Urol 2015;3:36-42.

5. Xiao KW, Zhou L, He Q, et al. Enucleation of the prostate for benign prostatic hyperplasia thulium laser versus holmium laser: a systematic review and meta-analysis. Lasers Med Sci 2019;34:815-26.

6. Li P, Wang C, Cao Q, et al. Prostatic Arterial Embolization Followed by Holmium Laser Enucleation of the Prostate as a Planned Combined Approach for Extremely Enlarged Benign Prostate Hyperplasia. Urol Int 2017;99:422-8.

7. Muhlstadt S, Friedl A, Mohammed N, et al. Five-year experience with the adjustable transobturator male system for the treatment of male stress urinary incontinence: a single-center evaluation. World J Urol 2017;35:145-51.

8. Altinbas NK, Hamidi N. Penile Doppler ultrasonography and elastography evaluation in patients with erectile dysfunction. Pol J Radiol 2018;83:e491-9.

9. Teo JS, Lee YM, Ho HSS. An update on transurethral surgery for benign prostatic obstruction. Asian J Urol 2017;4:195-8.

10. Muntener M, Aellig S, Kuettel R, et al. Sexual function after transurethral resection of the prostate (TURP): results of an independent prospective multicentre assessment of outcome. Eur Urol 2007;52:510-5.

11. Brunocilla E, Schiavina R, Borghesi M, et al. Preservation of the Internal Vesical Sphincter and Proximal Urethra During Retropubic Radical Prostatectomy may Improve Earlier Recovery of Continence in Selected Patients. Actas Urol Esp 2014;38:421-8.

12. Leong JY, Patel AS, Ramasamy R. Minimizing Sexual 
Dysfunction in BPH Surgery. Curr Sex Health Rep 2019;11:190-200.

13. Lim Ng K, Barber N. Prostatic hydroablation (Aquablation): A new effective ultrasound guided robotic waterjet ablative surgery for treatment of benign prostatic hyperplasia. Arch Esp Urol 2019;72:786-93.

14. Herrmann TRW, Gravas S, de la Rosette JJMCH, et al. Lasers in Transurethral Enucleation of the Prostate-Do We Really Need Them. J Clin Med 2020;9:1412.

15. Zhang J, Ou Z, Zhang X, et al. Holmium laser enucleation of the prostate versus thulium laser enucleation of the prostate for the treatment of large-volume prostates $>80 \mathrm{ml}$ : 18-month follow-up results. World J Urol 2020;38:1555-62.

16. Large T, Krambeck AE. Evidence-based outcomes of holmium laser enucleation of the prostate. Curr Opin Urol 2018;28:301-8.

17. Kim JK, Cho MC, Son H, et al. Patient Perception of Ejaculatory Volume Reduction After Holmium Laser Enucleation of the Prostate (HoLEP). Urology 2017;99:142-7.

18. Placer J, Salvador C, Planas J, et al. Effects of holmium laser enucleation of the prostate on sexual function. J Endourol 2015;29:332-9.

19. Kuebker JM, Miller NL. Holmium Laser Enucleation of the Prostate: Patient Selection and Outcomes. Curr Urol Rep 2017;18:96.

20. Oh SJ. Current surgical techniques of enucleation in holmium laser enucleation of the prostate. Investig Clin

Cite this article as: Li P, Wang C, Tang M, Han P, Meng X. Holmium laser enucleation of prostate by using en-bloc and bladder neck preservation technique: technical consideration and influence on functional outcomes. Transl Androl Urol 2021;10(1):134-142. doi: 10.21037/tau-20-852
Urol 2019;60:333-42.

21. Stolzenburg JU, Rabenalt R, Do M, et al. Intrafascial nerve-sparing endoscopic extraperitoneal radical prostatectomy. Eur Urol 2008;53:931-40.

22. Rieken M, Antunes-Lopes T, Geavlete B, et al. What Is New with Sexual Side Effects After Transurethral Male Lower Urinary Tract Symptom Surgery? Eur Urol Focus 2018;4:43-5.

23. Elshal AM, El-Assmy A, Mekkawy R, et al. Prospective controlled assessment of men's sexual function changes following Holmium laser enucleation of the prostate for treatment of benign prostate hyperplasia. Int Urol Nephrol 2017;49:1741-9.

24. Capogrosso P, Ventimiglia E, Ferrari M, et al. Longterm sexual outcomes after holmium laser enucleation of the prostate: which patients could benefit the most? Int J Impot Res 2016;28:189-93.

25. Klett DE, Tyson MD 2nd, Mmeje CO, et al. Patientreported sexual outcomes after holmium laser enucleation of the prostate: a 3 -year follow-up study. Urology 2014;84:421-6.

26. Maddox M, Pareek G, Al Ekish S, et al. Histopathologic changes after bipolar resection of the prostate: depth of penetration of bipolar thermal injury. J Endourol 2012;26:1367-71.

27. Orihuela E, Pow-Sang M, Motamedi M, et al. Mechanism of healing of the human prostatic urethra following thermal injury. Urology 1996;48:600-8. 